\title{
Imaging of pancreatic cancer using MDCT
}

Mohamed H. H. Ali ${ }^{a}$, Mohammad T. M. Solyman ${ }^{a}$, Mohammad Z. A. Murad ${ }^{a}$, Alaa E. H. Mohammed ${ }^{\text {b }}$

aDepartment of Radiodiagnosis, Sohag Faculty of Medicine, Sohag University. ${ }^{b}$ Department of General Surgery, Sohag Faculty of Medicine, Sohag University.

Introduction:Pancreatic cancer is one of the worst tumours in prognosis because it is usually already advanced when $1^{\text {st }}$ diagnosed, it is diagnosed late because of difficulty in diagnosis and detection as the pancreas is deeply located in the abdomen and the tumour is ill defined in most cases, when the tumour is located at the head, it obstructs the CBD is most cases causing severe jaundice, so pancreatic head tumours usually presents earlier than tumours at the pancreatic body or tail, early vascular invasion by the tumour also makes the tumourirresectable in most of the cases.

In this review article, the roles of imaging with $\mathrm{CT}$ in the detection and staging of pancreatic carcinoma will be discussed. The frequently employed techniques using these modality, the common imaging appearances of this tumor, and the limitations of imaging will be addressed.

Keywords: MDCT, CT angiography and pancreatic cancer.

\section{Introduction:}

Pancreatic adenocarcinoma is the most common nonendocrine malignancy of the pancreas and is the 4th leading cause of death is the United States. Most tumors arise in the head of the pancreas, and account for between 60 and $70 \%$ of cases (Kalra et al., 2002 ,Balci et al., 2001, Tamm et al., 2001 , Bluemke et al., 1995). Despite the recent advances in imaging and treatment, pancreatic adenocarcinoma continues to be a lethal disease. While newer diagnostic techniques have improved the accuracy for detecting these tumors, no significant inroads have been made in finding 'early' cancers. Most tumors are diagnosed late and approximately $85 \%$ of tumors are unresectable at the time of diagnosis. There are many reasons for this fact, but pancreatic carcinoma is unique in several respects:

(1) Symptoms manifest late

(2) Early extrapancreatic spread of tumor

(3) Rapid downhill course from diagnosis to death.

\section{Role of imaging}

The role of imaging in patients with suspected pancreatic carcinoma is:
(1) Confirm and stage tumor
(a) Determine if tumor is resectable or not

(b) Exclude pancreatic carcinoma in patients with symptoms suggestive of disease.

\section{TNM staging}

T0 No tumor

T1 Tumor confined to pancreas

T1a Tumor $2 \mathrm{~cm}$

T2 Tumor extension into duodenum, bile duct or peripancreatic tissues

T3 Tumor extension into stomach, colon, or adjacent great vessels

N0 No regional nodal metastases

N1 Regional nodal metastases

M0 No distant metastases

M1 Distal metastases.

While the criteria for unresectability vary from center to center, the presence of distant disease (metastases), local tumor extension, documented regional or distant lymph node metastases, and arterial invasion 
or encasement of major mesenteric arteries (celiac, hepatic, superior mesenteric artery) are generally accepted as criteria of unresectability. Venous involvement of the major mesenteric veins (superior mesenteric vein and portal vein) is not universally accepted as a criterion of unresectability as surgeons are performing en-block venous resection and venous reconstructions.

\section{Computed tomography techniques}

CT techniques in assessment of patients with suspected pancreatic carcinoma usually involve the use of thin section dynamic contrastenhanced helical CT obtained during the rapid bolus injection of large amounts of iodinated urographic contrast. The introduction of multidetector-row scanners has facilitated the acquisition of images during multiple phases of intravenous contrast administration. Utilization of a pancreatic parenchymal phase, using a scan delay of $40 \mathrm{~s}$ has resulted in superior pancreatic parenchymal enhancement. In some studies, this has led to superior tumor-to-parenchymal contrast differences, facilitating superior tumor detection, when compared to portal venous or delayed phases of imaging (Diehl et al., 1998; O'Malley et al.; 1999, Valls et al., 2002; Laghi et al., 2002; Prokesch et al., 2002). The information obtained from these multiphase exams was used to generate 3D images of the arterial, venous and pancreatico-biliary anatomy (Prokesch et al.; 2002,Catalano et al., 2003 ; Horton and Fishman, 2002 ; Johnson et al., 2003). These in select cases are useful for surgical planning.

\section{CT appearances}

Most pancreatic adenocarcinomas are of lower attenuation than the normally enhancing pancreatic parenchyma in all phases of contrast enhancement (Fig. 2). About $10 \%$ of pancreatic adenocarcinomas can be isoattenuating on CT. Pancreatic and bile duct dilatation are also common findings, as is atrophy proximal to the tumor. Perivascular tumor extension which leads to vascular involvement and arterial or venous encasement are also hallmarks of this tumor. In pancreatitis (either acute or chronic), they are usually streaky ill-defined areas of perivascular infiltration, whereas with pancreatic carcinoma it is usually seen as a 'cuff' of soft tissue encasing the peripancreatic vessels.

\section{CT: tumor detection and staging}

While CT is excellent in detecting unresectable tumors (>90\% accuracy), it frequently understages true tumor extent and even with early helical CT, the accuracy for assessing resectability was only around $70 \%$ (Bluemke et al., 1995). With the use of newer multislice helical CT scanners, tumor detection rates have improved to around $90-95 \%$. However only small improvements have been seen for determining resectability status. The most recent studies using multislice scanners have shown that positive predictive values for resectability are slightly above 80\% ( Diehl et al., 1998 ; Valls et al., 1997 ; Laghi et al., 2002). Reasons for this include the continued poor sensitivity for the detection of small peritoneal and liver metastases, metastases in normal sized lymph nodes and subtle peripancreatic tumor extension.

Predicting resectability based on tumor contact with peripancreatic vessels

Several CT studies have been performed to determine if the degree of contact of a tumor with the adjacent major peripancreatic arteries and veins 
could be used to predict if a tumor could be resected or not. These studies have shown that if there is a clear fat plane or normal pancreatic parenchyma interposed between the tumor and these vessels, in almost all instances the tumor is resectable. If there was tumor contact of $6180^{\circ}$, then the likelihood of resectability was high and if the degree of tumor contact was greater than $180^{\circ}$, it was most likely unresectable (Loyer et al., 1996 ; Lu DS et al., 1997) .

\section{Criteria for Arterial Involvement}

In the absence of obvious liver metastases or local tumor extension, tumor resectability will depend on the presence of vascular involvement. Involvement of important arteries (i.e., celiac axis, superior mesenteric artery, or splenic artery) will make surgical resection impossible. Isolated involvement of smaller branches such as the gastroduodenal artery will not preclude surgical resection as in. A CT grading system of vascular involvement has been reported by $\mathrm{Lu}$ et al. (Nakayama Y et al., 2001). These authors prospectively graded vessel involvement using a 0 - to 4-point scale based on circumferential contiguity of tumor to vessel and found that when more than $50 \%$ of the vessel circumference (grades 3 and 4) is in contact with a vessel, the tumor would not be resectable(Nakayama Yet al, 2001). This criterion resulted in a sensitivity and specificity for unresectability of $84 \%$ and $98 \%$, respectively (Nakayama $\boldsymbol{Y}$ et al., 2001).

Tumours at body or tail of the pancreas usually invades the splenic artery as ib (Fig 1).

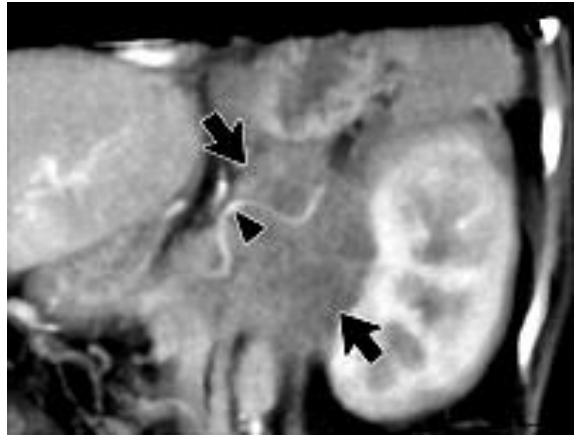

Fig. 1.63-year-old woman with pancreatic cancer. Coronal 3D volumerendered multidetector CT scan shows a pancreatic mass (arrows). In this orientation, mass is seen encasing splenic artery (arrowhead) (Karen $M$. Horton and Elliot K. 2002)

\section{Criteria for Venous Involvement:}

The CT grading system described by $\mathrm{Lu}$ et al. ( $\mathbf{L u}$ et al., 1997) can be applied to the evaluation of both arteries and veins. In that study, 25 patients with pancreatic adenocarcinoma underwent CT scanning in the pancreatic phase (40to 70 -sec delay) before surgery. These researchers prospectively graded arterial and venous involvement using a 0 - to 4-point scale based on contiguity of tumor with the adjacent vessel. When the tumor was in contact with more than $50 \%$ of the vessel circumference (grades 3 and 4), surgical resection was not possible $(\boldsymbol{L} \boldsymbol{u}$ et al., 1997). A similar study by O'Malley et al. (O'Malley et al, 1999). confirmed the results of Lu et al. $\mathrm{PV}$ is one of the large veins that are commonly invaded by pancreatic head tumours as in (Fig 2.), when invasion is more $50 \%$ of its circumference, the tumour will be irresectable. 


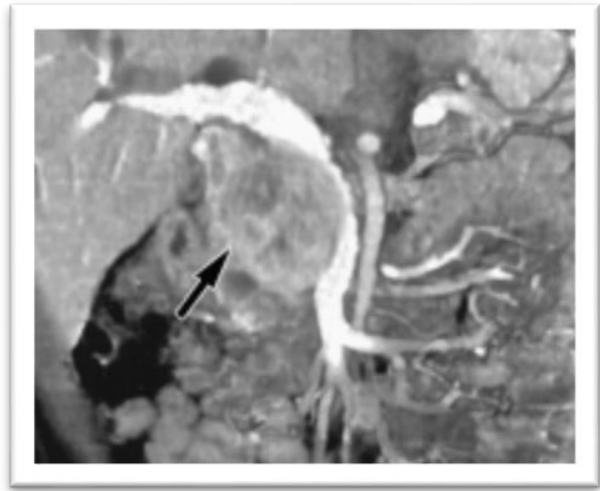

Fig. 2 : 48-year-old man with pancreatic cancer. Coronal volume rendered three-dimensional multidetector CT scan shows large mass (arrow) in head of pancreas. Mass is compressing and invading portal vein. Note bile ductal dilatation (Karen M. Horton and Elliot K. 2002).

\section{References:}

Balci N. C, andSemelka R. C, (2001). Radiologic diagnosis and staging of pancreatic ductal adenocarcinoma. European journal of radiology, 38(2), 105-112.

Horton K. M and Fishman E. K, (2002). Multidetector CT angiography of pancreatic carcinoma: part I, evaluation of arterial

involvement. AJR.American journal of roentgenology, 178(4), 827-831.

Johnson P. T, Heath, D. G, Hofmann L. V, Horton K. M, and Fishman E. K, (2003). Multidetector-row computed tomography with threedimensional volume rendering of pancreatic cancer: a complete preoperative staging tool using computed tomography angiography and volume-rendered cholangiopancreatography. Journal of computer assisted tomography, 27(3), 347-353.

Kalra M. K, Maher M. M, Sahani D. V, Digmurthy Sand Saini S, (2002). Current status of imaging in pancreatic diseases. Journal of computer assisted tomography, 26(5), 661675.

Loyer E. M, David C. L, DubrowR. A, Evans D. Band Charnsangavej C, (1996). Vascular involvement in pancreatic adenocarcinoma: reassessment by thin-section CT. Abdominal imaging, 21(3), 202-206.

Lu D. S, Reber H.A, Krasny R. M, Kadell B. Mand Sayre J, (1997). Local staging of pancreatic cancer: criteria for unresectability of major vessels as revealed by pancreatic-phase, thin-section helical CT. AJR. American journal of roentgenology, 168(6), 14391443.

Lu D. S, Reber H.A, Krasny R. M, Kadell B. M and Sayre J, (1997). Local staging of pancreatic cancer: criteria for unresectability of major vessels as revealed by pancreatic-phase, thin-section helical CT. AJR. American journal of roentgenology, 168(6), 14391443.

Nakayama Y, Yamashita Y, Kadota M, Takahashi M, Kanemitsu K, Hiraoka T, Hirota, M, Ogawa $M$ and Takeya $M$, 
(2001). Vascular encasement by pancreatic cancer: correlation of CT findings with surgical and pathologic results. Journal of computer assisted tomography, 25(3), 337-342.

O'Malley M. E, Boland G. W, Wood B. J, Fernandez-del Castillo C, Warshaw A. L and Mueller P. R, (1999). Adenocarcinoma of the head of the pancreas: determination of surgical unresectability with thin-section pancreatic-phase helical CT. AJR. American journal of roentgenology, 173(6), 1513-1518.

Prokesch R. W, Chow L. C, Beaulieu C. F, Nino-Murcia M, Mindelzun R. E, Bammer R, Huang J and Jeffrey R. B, (2002). Local staging of pancreatic carcinoma with multi-detector row CT: use of curved planar reformations initial experience. Radiology, 225(3), 759-765.

Valls C, Andía E, Sanchez A, Fabregat J, Pozuelo O, Quintero J. C, Serrano T, Garcia-Borobia, $F$ and Jorba R, (2002). Dualphase helical CT of pancreatic adenocarcinoma: assessment of resectability before surgery. AJR.American journal of roentgenology, 178(4), 821-826.

Diehl S. J, Lehmann K. J, Sadick M, Lachmann $\mathbf{R}$ and Georgi $M$, (1998). Pancreatic cancer: value of dual-phase helical CT in assessing resectability. Radiology, 206(2), 373-378.

Laghi A, Iannaccone R, Catalano C, Carbone I, Sansoni I, Mangiapane $\mathbf{F}$ and Passariello $\mathbf{R}$, (2002). Multislice spiral computed tomography in diagnosis and staging of pancreatic carcinoma: preliminary experience. Digestive and liver disease : official journal of the Italian Society of Gastroenterology and the Italian Association for the Study of the Liver, 34(10), 732-738.

Prokesch R. W, Chow L. C, Beaulieu C. F, Bammer $R$ and Jeffrey R. B, (2002). Isoattenuating pancreatic adenocarcinoma at multi-detector row CT: secondary signs. Radiology, 224(3), 764-768.

O'Malley M. E, Boland G. W, Wood B. J, Fernandez-del Castillo C, Warshaw A. L and Mueller P. R, (1999). Adenocarcinoma of the head of the pancreas: determination of surgical unresectability with thin-section pancreatic-phase helical CT. AJR. American journal of roentgenology, 173(6), 1513-1518.

Tamm E and Charnsangavej C, (2001). Pancreatic cancer: current concepts in imaging for diagnosis and staging. Cancer journal (Sudbury, Mass.), 7(4), 298-311.

Bluemke D. A, Cameron J. L, Hruban R. H, Pitt H. A, Siegelman S. S, Soyer and 
Fishman E. K, (1995). Potentially resectable pancreatic adenocarcinoma: spiral CT assessment with surgical and pathologic correlation. Radiology, 197(2), 381-385.

Catalano C, Laghi A, Fraioli F, Pediconi F, Napoli A, Danti M, Reitano I and Passariello $\mathbf{R}$, (2003). Pancreatic carcinoma: the role of high-resolution multislice spiral CT in the diagnosis and assessment of resectability. European radiology, 13(1), 149-156.

Horton, K. M and Fishman E. K, (2002).Multidetector angiography of pancreatic carcinoma: part I, evaluation of arterial involvement. AJR. American journal of roentgenology, 178(4), 827-831.

Horton, K. M and Fishman E. K, (2002). Multidetector CT angiography of pancreatic carcinoma: part 2, evaluation of venous

involvement. AJR.American

journal of roentgenology, 178(4), 833-836. 